\title{
TRIVIAL EXTENSION OF A RING WITH BALANCED CONDITION
}

\author{
HIDEAKI SEKIYAMA
}

\begin{abstract}
A ring $R$ is called QF-1 if every faithful $R$-module is balanced. In this paper we study commutative QF-1 rings. It is shown that a commutative QF-1 ring is local if and only if it is uniform. It is well known that commutative artinian QF-1 rings are QF, but Osofsky has constructed a nonartinian nonnoetherian commutative injective cogenerator, so QF-1, ring which is a trivial extension of a valuation ring. It is shown that if a trivial extension of a valuation ring is QF-1, then it has a nonzero socle. Furthermore such rings become injective cogenerator rings under certain conditions.
\end{abstract}

Throughout this paper rings are commutative rings with unity and modules are unital. Hence the trivial extension of a ring $B$ by a $B$-module $E$ is defined to be the ring whose additive group is the direct sum $B \oplus E$ with multiplication given by

$$
(b, e) \cdot\left(b^{\prime}, e^{\prime}\right)=\left(b b^{\prime}, b^{\prime} e+b e^{\prime}\right) .
$$

An $R$-module $M$ is called balanced if the canonical ring homomorphism of $R$ into the double centralizer of $M$ is surjective, and a ring $R$ is said to be QF-1 if every faithful $R$-module is balanced. A ring $R$ is said to be PF if $R$ is an injective cogenerator as an $R$-module.

There is an interesting theorem on QF-1 rings that a commutative QF-1 artinian ring $R$ is QF (V. P. Camillo [1] and S. E. Dickson and K. R. Fuller [3]) which has been generalized by C. M. Ringel [10], H. H. Storrer [12] and $H$. Tachikawa [14] under the weaker condition that $R$ is noetherian or perfect instead of the assumption on $R$ to be artinian.

On the other hand B. L. Osofsky [9, Example 1] has constructed a commutative nonnoetherian PF ring which is a trivial extension of a valuation ring. It is known that $\mathrm{PF}$ rings are $\mathrm{QF}-1$, so her example distinguishes commutative QF-1 rings from QF rings. Hence trivial extension QF-1 rings of valuation rings are worthy to be considered. The purpose of this paper is to show that such a ring has a nonzero socle, which is an important necessary condition for a ring to be PF, and that it is PF under certain conditions.

V. P. Camillo [2] and H. H. Storrer [11] have proved that a commutative QF-1 ring $R$ has the principal extension property which plays the important

Received by the editors May 25, 1978.

AMS (MOS) subject classifications (1970). Primary 13A15, 13H99, 16A36, 16A52; Secondary 13B99, 16A34.

Key words and phrases. QF-1 ring, double centralizer, trivial extension, valuation ring, injective cogenerator. 
role in this paper, that is, every homomorphism of any principal ideal of $R$ into $R$ can be extended to all of $R$. Furthermore M. Ikeda and T. Nakayama [7] have proved that if a ring $R$ has the principal extension property, then every principal ideal of $R$ satisfies the annihilator condition, that is,

$$
\operatorname{Ann}_{R} \operatorname{Ann}_{R}(R r)=R r \text { for all } r \text { in } R \text {. }
$$

Although C. M. Ringel has proved in [10] that if a commutative QF-1 ring has a nonzero socle and if it is local then it is uniform, we shall prove it by using the principal extension property without assuming the existence of a nonzero socle and prove the converse of it.

THEOREM 1. A commutative $Q F-1$ ring is local if and only if it is uniform.

Proof. At first we shall consider the case where a local commutative QF-1 ring $R$ has a nontrivial socle. Let $S$ be a minimal ideal. For an arbitrary nonzero element $x$ in $R$, an $R$-module $R x /(\operatorname{Rad}(R)) x$ is simple and isomorphic to $S$. Thus there exists a nonzero $R$-homomorphism of $R x$ into $R$ whose image is $S$. The principal extension property means $S \subset R x$. So $R$ is uniform since $S$ is essential in $R$.

Next we shall consider a local commutative QF-1 ring $R$ with zero socle. In this case it is to be noted that the maximal ideal $W(=\operatorname{Rad}(R))$ of $R$ is faithful. We shall prove the fact that if $x$ and $y$ are nonzero elements of $R$ with $R x \cap R y=0$, then $\operatorname{Ann}_{R}(x)+\operatorname{Ann}_{R}(y)=R$. This implies either $\operatorname{Ann}_{R}(x)=R$ or $\operatorname{Ann}_{R}(y)=R$, because $R$ is assumed to be local. Hence either $x$ or $y$ is zero, which contradicts the assumption that $x$ and $y$ are nonzero elements. So suppose that $\operatorname{Ann}_{R}(x)+\operatorname{Ann}_{R}(y) \neq R$. Then $W$ contains $\operatorname{Ann}_{R}(x)+\operatorname{Ann}_{R}(y)$, and an $R$-module $R / W x \oplus R / W y$ is faithful and so balanced, since $W x \cap W y \subset R x \cap R y=0$. We can define a nonzero map $\phi$ of $R / W x \oplus R / W y$ into $R / W x \oplus R / W y$ as follows $(a, b) \mapsto(x a, y b)$ for $a \in R / W x, b \in R / W y$, since $R x \neq W x$ and $R y \neq W y$. Every element in $\operatorname{Hom}_{R}(R / W x, R / W y)$ is induced by some element $r$ of $R$ such that $W x r \subset$ $W y$, so $r$ annihilates $x$ since $W x \cap W y=0$ and $W$ is faithful. Also since $W y x \subset W y \cap W x=0$, we have $\operatorname{Ann}_{R}(x) \supset W y$. Consequently we have

$$
T(R / W x, R / W y)=\operatorname{Ann}_{R}(x) / W y,
$$

where

$$
T(R / W x, R / W y)=\sum\left\{\operatorname{Im}(f) ; f \in \operatorname{Hom}_{R}(R / W x, R / W y)\right\} .
$$

Thus $(x-y) T(R / W x, R / W y)=0$ since $\operatorname{Ann}_{R}(x) \subset W$. Similarly $(y-$ $x) T(R / W y, R / W x)=0$. Then by the Camillo criterion [1, 11. Lemma], $\phi$ is an element of the double centralizer of $R / W x \oplus R / W y$. So it should be induced by the multiplication with an element $r$ of $R$. But then the element $(1+W x, 1+W y)$ in $R / W x \oplus R / W y$ is mapped onto $(r+W x, r+W y)$ $=(x+W x, y+W y)$, thus $r-x \in W x$ and $r-y \in W y$, and therefore $r \in R x \cap R y=0$, a contradiction.

Conversely, suppose that a commutative QF-1 ring $R$ is uniform. We fix an arbitrary nonunit element $x$ of $R$. For any element $r$ of $R$, the element $r x$ is a 
nonunit. If $\operatorname{Ann}_{R}(r x)=0$, a map of $R r x$ into $R$ as follows $r^{\prime} r x \mapsto r^{\prime}$ for $r^{\prime} \in R$ is a well-defined $R$-homomorphism. By the principal extension property, there is an element $p \in R$ such that $p r x=1$, which contradicts the fact that $r x$ is a nonunit. Thus we have $\operatorname{Ann}_{R}(r x) \neq 0$. Since $R$ is uniform and $\operatorname{Ann}_{R}(r x) \cap \operatorname{Ann}_{R}(1-r x)=0$, we have $\operatorname{Ann}_{R}(1-r x)=0$, so $1-r x$ is a unit by the above argument. We have showed that every nonunit element is in the radical of $R$, which implies that $R$ is local.

V. P. Camillo has proved in [1, Lemma 2] that if the direct sum of a faithful $R$-module $M$ and a simple $R$-module $S$ is balanced then either $\operatorname{Hom}_{R}(S, M)$ or $\operatorname{Hom}_{R}(M, S)$ is not zero. Hence it follows that if $R$ is QF-1 and if a faithful $R$-module $M$ has a zero socle, then the (Jacobson) $\operatorname{radical} \operatorname{Rad}(M)$ of $M$ is not equal to $M$. We note that for a module $M$ over a local ring $R$, $\operatorname{Rad}(M)$ is equal to $\operatorname{Rad}(R) \cdot M$.

We need some definitions. A module $E$ is called uniserial if the lattice of submodules of $E$ is linearly ordered by inclusion, and a ring $B$ is called a valuation ring if $B$ is uniserial as a $B$-module. A valuation ring $B$ is said to be maximal if every system of pairwise solvable congruences of the form

$$
x \equiv x_{\alpha}\left(I_{\alpha}\right) \quad\left(\alpha \in A, x_{\alpha} \in B, I_{\alpha} \text { an ideal of } B\right)
$$

has a simultaneous solution in $B$. We say $B$ is almost maximal if the above congruences have a simultaneous solution whenever $\bigcap_{\alpha \in A} I_{\alpha} \neq 0$ (cf. C. Faith [4]).

Now, we are ready to prove our next theorem.

THEOREM 2. Let $R$ be the trivial extension ring of a valuation ring $B$ by $a$ nonzero $B$-module $E$. If $R$ is $Q F-1$, then the following hold:

(a) $E$ is faithful and uniserial;

(b) the socle of $R$ is equal to $\left(0, \operatorname{Soc}_{B}(E)\right)$ and is not zero.

Proof. (a) $R$ is a commutative local ring with the maximal ideal $(\operatorname{Rad}(B), E)$, and $R$ is uniform by Theorem $1 .\left(\operatorname{Ann}_{B}(E), 0\right)$ and $(0, E)$ are ideals with zero intersection and so, by the uniformness of $R, A_{B} n_{B}(E)$ must be zero, that is, $E$ is faithful.

For the second assertion it is sufficient to show that for any two elements $x, y$ of $E$, it holds that either $B x \subset B y$ or $B x \supset B y$. Since $B$ is a valuation ring, we may assume $\operatorname{Ann}_{B}(y)$ contains $\operatorname{Ann}_{B}(x)$. Then

$$
\operatorname{Ann}_{R}(0, x)=\left(\operatorname{Ann}_{B}(x), E\right) \subset\left(\operatorname{Ann}_{B}(y), E\right)=\operatorname{Ann}_{R}(0, y)
$$

Two principal ideals $R(0, x)=(0, B x)$ and $R(0, y)=(0, B y)$ satisfy the annihilator condition, so we have

$$
(0, B x)=\operatorname{Ann}_{R} \operatorname{Ann}_{R}(0, x) \supset \operatorname{Ann}_{R} \operatorname{Ann}_{R}(0, y)=(0, B y),
$$

which shows that $B x \supset B y$.

(b) Assume that there exists an element $(b, e)$ of $R$ which generates a minimal ideal such that $b \neq 0$. Noting that $b E \neq 0$, we have $R(b, e) \supsetneqq$ $(0, b E) \neq 0$, which contradicts the minimality of $R(b, e)$. Thus $\operatorname{Soc}_{R}(R)$ must be $\left(0, \operatorname{Soc}_{B}(E)\right)$. 
Next we must show that $R$ has a nonzero socle. If $\operatorname{Soc}_{B}(B)$ is not zero, then we have a minimal ideal $(0, B b e)$, where $b$ is a generator of a minimal ideal of $B$ and $e$ is an element of $E$ such that $b e \neq 0$.

Thus we may assume $\operatorname{Soc}_{B}(B)=0$. Let $W$ be the radical of $B$. It is to be noted that $W$ is a faithful ideal of $B$.

We claim that $E$ is not a cyclic $B$-module. Suppose $E$ is cyclic with a generator $e$, then the ideal ( $W, W e$ ) of $R$ is faithful and has a zero socle. Then we have $(W, W e) \neq \operatorname{Rad}(W, W e)=\left(W^{2}, W e\right)$. Hence $W$ is generated by one element $w$ since $B$ is a valuation ring. Then

$$
(0, W e)=\operatorname{Ann}_{R} \operatorname{Ann}_{R}(0, w e)=\operatorname{Ann}_{R}(0, E)=(0, E),
$$

which contradicts $E \neq W e$.

Next, we claim that any proper submodule $F$ of $E$ is not faithful. We can take two elements $e_{1}, e_{2}$ of $E$ so that

$$
F \subset B e_{1} \varsubsetneqq B e_{2} \subset E .
$$

From the proof of (a), $\operatorname{Ann}_{B}\left(e_{1}\right)$ contains strictly $\operatorname{Ann}_{B}\left(e_{2}\right)$; specifically $\operatorname{Ann}_{B}\left(e_{1}\right)$ is nonzero, so is $\operatorname{Ann}_{B}(F)$.

Now assume $\operatorname{Soc}_{R}(R)=0$. Then the radical $(W, E)$ of $R$ is faithful and has a zero socle, so that $(W, E) \neq \operatorname{Rad}(W, E)=\left(W^{2}, W E\right) . W E$ is a faithful $B$-module, so it is equal to $E$. Thus $W$ is generated by one element $w$. $\operatorname{Rad}(R)=R(w, 0)$ leads to $R$ having a nonzero socle by applying the proof of C. M. Ringel [10, Lemma 3], a contradiction. This completes the proof.

COROllary 3. Let $R$ be the trivial extension ring of a valuation ring $B$ of an injective nonzero $B$-module $E$. If $R$ is $Q F-1$, then the following hold.

(a) $E$ is the injective hull of $B / \operatorname{Rad}(B)$, so $B$ is an almost maximal valuation ring;

(b) if $E$ is cyclic, then $B$ and $R$ are $P F$;

(c) if $B$ is not an integral domain, then $R$ is $P F$.

Proof. (a) We know that $\left(0, \operatorname{Soc}_{B}(E)\right)$ is minimal and essential in $R$ from Theorems 1 and 2. So $\operatorname{Soc}_{B}(E)$ is simple and essential in $E$, and thus $E$ is the injective hull of $B / \operatorname{Rad}(B)$. By C. Faith [4, Theorem 20.49], $B$ is almost maximal.

(b) If $E$ is cyclic, $E$ is isomorphic to $B$. By (a), $E$ is an injective cogenerator, so is $B$. By B. J. Müller [8, Theorem 10], then $R$ is PF.

(c) Since $B$ is an almost maximal nonintegral domain, $B$ is maximal by $C$. Faith [4, Proposition 20.46]. We shall show that the endomorphism ring of $E$ is canonically isomorphic to $B$. This implies that $R$ is injective by $R$. M. Fossum et al. [6, Corollary 4.37] since $E$ is injective. Now let $f$ be any element of the endomorphism ring of $E$, and $\left\{e_{\alpha}\right\}_{\alpha \in A}$ be a set of generators of $E$. For every $e_{\alpha}$, a map of $R\left(0, e_{\alpha}\right)$ into $R$, as follows $r\left(0, e_{\alpha}\right) \mapsto\left(0, b f e_{\alpha}\right)$ for $r=(b, e)$ $\in R$, is a well-defined $R$-homomorphism, so there exists an element $b_{\alpha}$ of $B$ such that $f e_{\alpha}=b_{\alpha} e_{\alpha}$ by the principal extension property. Then we consider 
the system of congruences as follows:

$$
x \equiv b_{\alpha}\left(I_{\alpha}\right) \quad\left(\alpha \in A, b_{\alpha} \in B, I_{\alpha}=\operatorname{Ann}_{B}\left(e_{\alpha}\right)\right) .
$$

For any $\alpha$ and $\beta$ in $A$, we may assume that $B e_{\alpha} \subset B e_{\beta}$ since $E$ is uniserial. If $e_{\alpha}=b e_{\beta}$ by an appropriate element $b$ of $B$, then $b_{\beta} e_{\alpha}=b b_{\beta} e_{\beta}=b f e_{\beta}=f e_{\alpha}$ $=b_{\alpha} e_{\alpha}$, so $b_{\beta}-b_{\alpha} \in I_{\alpha}$. This shows that the above system is pairwise solvable. There exists a solution of it, since $B$ is maximal. This solution induces $f$. This completes the proof.

C. Faith [5, Theorem 6A] has given equivalent conditions on a trivial extension of a ring to be a PF valuation ring. Here we shall give the necessary and sufficient condition in order that a trivial extension QF-1 ring is a valuation ring.

Corollary 4. Let $R$ be the trivial extension $Q F-1$ ring of a ring $B$ by $a$ nonzero $B$-module $E$. Then the following are equivalent:

(a) $R$ is a valuation ring;

(b) $B$ is an integral domain and is a valuation ring.

Proof. We assume (a). For any ideals $I, J$ of $B,(I, I E),(J, J E)$ are ideals of $R$. We have either $(I, I E) \supset(J, J E)$ or $(I, I E) \subset(J, J E)$, so either $I \supset J$ or $I \subset J$, which means that $B$ is a valuation ring.

Then $E$ is faithful by Theorem 2. Next, take any nonzero element $b$ of $B$. An ideal of $R$ generated by $(b, 0)$ is $(B b, b E)$, which is not contained in an ideal $(0, E)$ of $R$. Thus it contains $(0, E)$, hence we have $b E=E$. The regularity of $b$ follows from the faithfulness of $E$.

We assume (b). By Theorem 2, $E$ is uniserial and faithful. We are in the case where $\operatorname{Soc}_{B}(B)=0$ because $B$ is an integral domain. From the proof of Theorem 2, any proper submodule of $E$ is not faithful. Take an ideal $I$ of $R$ such that $I$ contains one element $(b, e)$ with $b \neq 0$. Then a submodule $b E$ of $E$ is faithful, so equal to $E$, which leads to $I$ containing $(0, E)$. Thus the ideals of $R$ are of the form $(J, E)$, with $J$ an ideal of $B$, and of the form $(0, F)$, with $F$ a submodule of $E$. Since both $B$ and $E$ are uniserial, $R$ is a valuation ring.

Acknowledgement. The author wishes to express his best thanks to Professor H. Tachikawa for his helpful suggestions and encouragements.

\section{REFERENCES}

1. V. P. Camillo, Balanced rings and a problem of Thrall, Trans. Amer. Math. Soc. 149 (1970), 143-153. MR 41 \#5417.

2. A property of $Q F-1$ rings (preprint).

3. S. E. Dickson and K. R. Fuller, Commutative $Q F-1$ artinian rings are $Q F$, Proc. Amer. Math. Soc. 24 (1970), 667-670. MR 40 \#646.

4. C. Faith, Algebra. II. Ring Theory, Die Grundlehren der Math. Wissenchaften, no. 191, Springer-Verlag, Berlin and New York, 1976. MR 55 \#383.

5. C. Faith, Self-injective rings (preprint).

6. R. M. Fossum, P. A. Griffith and I. Reiten, Trivial extensions of abelian categories, Lecture Notes in Math., vol. 456, Springer-Verlag, Berlin and New York, 1975. MR 52 \# 10810.

7. M. Ikeda and T. Nakayama, On some characteristic properties of quasi-Frobenius and regular rings, Proc. Amer. Math. Soc. 5 (1954), 15-19. MR 15, 677.

8. B. J. Müller, On Morita duality, Canad. J. Math. 21 (1969), 1338-1347. MR 41 \#258. 
9. B. L. Osofsky, A generalization of QF rings, J. Algebra 4 (1966), 373-387; erratum, ibid. 9 (1968), 120. MR 34 \# 4305; MR 36 \#6443.

10. C. M. Ringel, Commutative $Q F-1$ rings, Proc. Amer. Math. Soc. 42 (1974), 365-368. MR 49 \#9022.

11. H. H. Storrer, Epimorphismen von kommutativen Ringen, Comment. Math. Helv. 43 (1968), 378-401. MR 39 \#4137.

12. __ Epimorphic extensions of noncommutative rings, Comment. Math. Helv. 48 (1973), 72-86. MR 48 \#342.

13. H. Tachikawa, Quasi-Frobenius rings and generalizations, Lecture Notes in Math., vol. 351, Springer-Verlag, Berlin and New York, 1973. MR 50 \#2233.

14. __ Commutative perfect $Q F$-1 rings, Proc. Amer. Math. Soc. 68 (1978), 261-264.

15. R. M. Thrall, Some generalizations of $Q F$-algebras, Trans. Amer. Math. Soc. 64 (1948), 173-183. MR 10, 98.

Institute of Mathematics, University of Tsukuba, SAKURA-MURa Nimhari-Gun Ibaraki, 300-31 JAPAN 